\section{Caracterização do uso de medicamentos entre idosos de uma unidade do Programa Saúde da Família}

\author{
Use of medicines by the elderly in a Family Health \\ Program unit in Brazil
}

\author{
${ }^{1}$ Faculdade de Medicina de \\ Marília, Marília, Brasil. \\ 2 Programa de Pós- \\ Graduação em Ciências da \\ Saúde, Universidade Federal \\ de São Paulo, São Paulo, \\ Brasil. \\ 3 Escola Paulista de \\ Medicina, Universidade \\ Federal de São Paulo, \\ São Paulo, Brasil. \\ 4 Secretaria Municipal de \\ Higiene e Saúde de Marília, \\ Marília, Brasil. \\ Correspondência \\ M. J. S. Marin \\ Faculdade de Medicina de \\ Marília. \\ Av. Brigadeiro Eduardo \\ Gomes 1886, Marilia, SP \\ 17514-000, Brasil. \\ marnadia@terra.com.br
}

\begin{abstract}
The use of multiple medicines is frequent among the elderly. Although such practice is usually necessary, it predisposes to risks of adverse events and drug interaction. The present study was intended to verify the characteristics of medication among the elderly in relation to socio-demographic profile, the most commonly used drug classes, and treatment adherence. This was a descriptive study that included 301 elderly in the coverage area of a Family Health Program, using a previously structure home interview. The sample was predominantly female, with a high illiteracy rate and alterations in cognitive status and various forms of dependency. The subjects showed an average of 2.5 diagnoses and used 2.9 drugs per individual. Some of the drugs are classified as high-risk for the age bracket. Treatment adherence data showed dependence on others for administration, self-medication, forgetfulness in relation to medication, and insufficient knowledge of the drugs taken. Systematic followup is needed for elderly persons using multiple medicines, in addition to encouragement for non-pharmaceutical measures and an emphasis on educational measures for lifestyle change, backed by a multidisciplinary team.
\end{abstract}

Drug Utilization; Aged; Family Health Program

\author{
Maria José Sanches Marin 1,2 \\ Luiz Carlos de Oliveira Cecílio 3 \\ Alexandre Eduardo W. Ugolini Ferrazoli Perez 4 \\ Fernando Santella 1 \\ Camila Batista Andrade Silva 1 \\ José Roberto Gonçalves Filho 1 \\ Lidiane Cola Roceti 1
}

\section{Introdução}

Nos últimos decênios tem aumentado a preocupação com melhoria das condições de vida na velhice. No Brasil, a população idosa, em 2002, perfazia um total de 14,1 milhões de pessoas e projetava-se para 2025 um total de 33,4 milhões, sendo que entre 1950 e 2025 a população idosa terá crescido 16 vezes contra 5 vezes a população total ${ }^{1}$. Destaca-se na população idosa o aumento de pessoas com oitenta anos ou mais, sendo essa a faixa etária de maior crescimento, tanto nos países desenvolvidos como nos países em desenvolvimento 2 .

Juntamente com as modificações da estrutura etária da população, constatam-se mudanças epidemiológicas, com a substituição das causas principais de morte por doenças parasitárias, de caráter agudo, pelas doenças crônico-degenerativas (diabetes, acidente vascular cerebral, neoplasias, hipertensão arterial, demência senil e outras), que se transformam em problemas de longa duração e envolvem, para atendimento adequado, grande quantidade de recursos materiais e humanos 3

Por conviver com problemas crônicos de saúde, os idosos utilizam com freqüência os serviços de saúde e são consumidores de grande número de medicamentos 4, que embora necessários em muitas ocasiões, quando mal utilizados podem desencadear complicações sérias para a saúde e aumento dos custos individuais e governamen- 
tais 5 . Acrescenta-se que devido aos inegáveis ganhos terapêuticos obtidos com o uso dos produtos farmacêuticos, eles passaram a ser utilizados de forma indiscriminada e irracional, seguindo uma lógica de mercado.

Nos Estados Unidos estima-se que de $25 \%$ a $32 \%$ de todos os medicamentos são consumidos por idosos que representam $12 \%$ da população. Os efeitos adversos das drogas são 2,5 vezes mais freqüentes nos idosos do que na população de outra faixa etária 6 . Em torno de $28,2 \%$ do gasto total com internações hospitalares, nos Estados Unidos, deve-se à morbidade e mortalidade resultantes do uso inadequado de drogas 7.

No Brasil, estudo baseado na Pesquisa Nacional por Amostra de Domicílios (PNAD) aponta que $50 \%$ dos idosos têm renda pessoal menor que um salário mínimo e o gasto médio mensal com medicamentos compromete aproximadamente um quarto da renda ${ }^{8}$.

Acrescenta-se que os idosos constituem 50\% das pessoas que utilizam múltiplos medicamentos. Além disso, é comum encontrar, em suas prescrições, doses e indicações inadequadas, interações medicamentosas, associações e redundância, além do uso de medicamentos sem valor terapêutico 9 .

A complexidade dos esquemas medicamentosos, juntamente com a falta de entendimento, esquecimento, diminuição da acuidade visual e destreza manual que ocorrem no idoso, contribui para que haja grande quantidade de erros na administração de medicamentos 10. Além disso, acrescenta-se, em nossa realidade, alto índice de analfabetismo, o que pode comprometer o entendimento e levar ao uso incorreto do medicamento 11,12 .

A Organização Mundial da Saúde (OMS) considera que mais de $50 \%$ dos medicamentos são prescritos ou dispensados de forma inadequada e que $50 \%$ dos pacientes tomam medicamentos de maneira incorreta levando a alto índice de morbidade e mortalidade. Acrescenta que os tipos mais comuns de uso irracional de medicamentos estão relacionados às pessoas que utilizam polifarmácia, ao uso inapropriado de antibiótico e de medicamento injetável, a automedicação e a prescrição em desacordo com as diretrizes clínicas 13 .

Além de todas as dificuldades que os idosos apresentam ao fazer uso de medicamentos, eles podem também não aderir ao mesmo, o que torna a situação ainda mais complexa. A adesão é considerada um processo multifatorial que se estrutura em uma parceria entre quem cuida e quem é cuidado e diz respeito à freqüência, à constância e à perseverança em relação aos cuidados necessários para quem vive algum problema de saúde.
A adesão fica mais comprometida em situações que requerem tratamentos longos e quando há necessidade de alteração no estilo de vida, o que é freqüente entre os idosos 14 . Destaca-se ainda que a adesão depende da consciência cidadã e cidadania "não é algo natural", posto que está submetida a regras sociais e culturais 15 .

Esse aspecto representa um desafio aos profissionais da equipe de saúde que muitas vezes adotam condutas autoritárias, considerando o saber científico como verdade absoluta. Tal postura talvez explique por que, em um contexto de evidente transição demográfica e epidemiológica, os modelos de atenção à saúde não venham conseguindo resultados satisfatórios 16 .

Considerando a importância que representa o uso correto de medicamentos entre a população idosa e a necessidade de se adotarem estratégias que visem à adesão ao tratamento e à utilização correta dos medicamentos, propomos, para o presente estudo, os seguintes objetivos: (i) caracterizar o perfil sócio-demográfico de idosos pertencentes à área de cobertura de uma unidade de saúde da família; (ii) verificar as classes medicamentosas mais utilizadas; (iii) verificar a adesão ao uso dos medicamentos prescritos.

\section{Metodologia}

Trata-se de um estudo transversal e descritivo realizado a partir de inquérito no domicílio dos idosos residentes na área de abrangência de uma unidade de saúde da família, de uma cidade do interior paulista.

A população de referência foi constituída pelo universo de idosos residentes na área citada ( $\mathrm{n}=461)$. A identificação deu-se por meio do cadastro dos agentes comunitários de saúde. Participaram efetivamente do estudo 301 (65,3\%) idosos. Entre os demais, 96 (20,8\%) recusaramse a participar, 61 (13,2\%) não foram encontrados após a segunda visita e três eram portadores de doenças mentais, encontrando-se sozinhos no domicílio no momento da visita. A alta incidência de idosos que se recusaram a participar do estudo pareceu-nos poder ser explicada pela extensão do instrumento, o qual visava uma avaliação geriátrica ampla, com a finalidade de identificar as necessidades de saúde e propor, de forma articulada com a equipe da unidade, um plano de intervenção. Alguns idosos chegavam a iniciar a entrevista, mas desistiam ou apresentavam sinais de inquietação, o que foi respeitado pelos pesquisadores.

O instrumento de avaliação utilizado permite fazer a caracterização sócio-demográfica dos idosos, além de suas condições de comunicação, 
de utilização de serviços de saúde, da existência ou não de cuidador, do diagnóstico médico referido, além do grau de dependência e do estado cognitivo. Considerou-se que as condições de comunicação estavam preservadas quando o idoso tinha condições de fornecer todas as informações; parcialmente comprometidas, quando precisava de ajuda para fornecer as informações; e comprometidas, quando não foi possível coletar qualquer informação diretamente com o entrevistado. O grau de dependência foi verificado a partir de uma escala composta por 12 indicadores de dependência que avaliam as atividades instrumentais (fazer compras, pegar ônibus, administrar as finanças e tomar os medicamentos adequadamente) e as atividades básicas (continência, vestir-se, tomar banho, comer, pentearse, ir ao banheiro, passar para cama e sair dela) da vida diária, organizadas hierarquicamente. De acordo com as 12 atividades de vida diária citadas, os idosos são classificados em quatro categorias: (1) independente total - idoso capaz de realizar todas as atividades; (2) dependente leve - idoso que necessita de ajuda para realizar de uma a três atividades de vida diária; (3) dependente moderado - idoso que necessita de ajuda para realizar de quatro a seis atividades de vida diária; e (4) dependente severo - aquele que necessita de ajuda para realizar sete e/ou mais dessas atividades 17 . A capacidade cognitiva foi verificada por meio do Mini-Exame do Estado Mental (MEEN) de Folstein padronizado para a população brasileira, com a finalidade de caracterizar a presença de prejuízos cognitivos 18. Além disso, realizou-se uma adaptação do roteiro para avaliar a adesão ao tratamento medicamentoso proposto por Burguez-Diaz 19, com questões sobre medicamentos que utiliza, conhecimento sobre a indicação e efeitos colaterais dos mesmos, administração, aquisição, uso de automedicação, conhecimento sobre os medicamentos e dificuldades relacionadas ao seu uso.

Para a identificação dos medicamentos, foram considerados aqueles de que o idoso estava fazendo uso no dia da entrevista, incluindo os prescritos e os não prescritos. Para isso, foi solicitado ao idoso que trouxesse à vista do entrevistador os medicamentos utilizados. Os medicamentos foram classificados de acordo com Anatomical Therapeutic Chemical Code (ATC) adotada pela OMS 13. Nesta classificação, eles são divididos de acordo com o grupo anatômico ou sistema em que atuam e suas propriedades químicas, terapêuticas e farmacológicas. Para identificar as substâncias a partir dos nomes comerciais, empregou-se o Dicionário de Especialidade Farmacêutica (DEF) (2005/2006) 20.
Foi verificado, ainda, se os medicamentos utilizados estavam com o nome genérico e se pertenciam à lista de medicamentos selecionados pela Secretaria Municipal de Higiene e Saúde de Marília 21.

Para realização do estudo houve autorização do Secretário da Saúde do município e da equipe de saúde da unidade. Participaram os idosos que, após receber os esclarecimentos, estavam de acordo e assinaram o Termo de Consentimento Livre e Esclarecido, aprovado pelo Comitê de Ética e Pesquisa com Seres Humanos da Faculdade de Medicina de Marília. A coleta de dados foi realizada no domicílio dos idosos, por estudantes dos cursos de enfermagem e medicina.

\section{Resultados}

Na Tabela 1, constata-se que $186(61,8 \%)$ dos idosos entrevistados são do sexo feminino e 115 $(38,2 \%)$ do sexo masculino, com predomínio de idosos na faixa etária entre 60 e 69 anos de idade. Quanto à escolaridade, dois terços deles, 207 (68,1\%), são analfabetos ou têm o primeiro grau incompleto.

Destaca-se, quanto ao estado marital, que 111 (36,9\%) vivem sem o companheiro. Quanto aos serviços de saúde, $188(62,4 \%)$ utilizam apenas a unidade de saúde da família, os demais, $58(19,2 \%)$, apenas o convênio de saúde e $52(17,3 \%)$ utilizam tanto a unidade de saúde da família como os serviços do convênio. Além disso, a maioria dos idosos, 268 (89,6\%), apresenta boas condições de comunicação. Entre eles, 99 (32,9\%) moram com o companheiro/ filhos e netos, $91(30,2 \%)$ apenas com o companheiro e 30 (10\%) residem sozinhos. Em caso de necessidade, são os filhos que prestam cuidados para mais da metade deles, 155 (51,5\%), e o companheiro, para $103(34,2 \%)$ dos idosos entrevistados.

Dos 301 (100\%) idosos entrevistados, 224 $(74,4 \%)$ apresentaram escores considerados normais para o estado cognitivo. Quase dois terços são independentes para as atividades de vida diária, enquanto 63 (20,9\%) são dependentes leves, $25(8,3 \%)$ dependentes moderados e $14(4,7 \%)$ dependentes severos.

Na Tabela 2, encontram-se os diagnósticos referidos pelos idosos entrevistados, agrupados de acordo com a Classificação Internacional de Doenças, 10a revisão (CID-10) 22. Os 301 idosos entrevistados referiram 746 doenças, com uma média de 2,5 diagnóstico/idoso e as doenças mais referidas são aquelas do sistema circulatório, 328 (44\%). Seguem-se a estas, as doenças do sistema osteomuscular e do tecido conjuntivo, 
Tabela 1

Distribuição dos idosos entrevistados de acordo com a caracterização sócio-demográfica, estado cognitivo e grau de dependência. Marília, São Paulo, Brasil, 2007.

\begin{tabular}{|c|c|c|}
\hline \multirow{2}{*}{$\begin{array}{l}\text { Características sócio-demográficas, grau de } \\
\text { estado de dependência e estado cognitivo }\end{array}$} & \multicolumn{2}{|c|}{ Total } \\
\hline & $\mathrm{n}$ & $\%$ \\
\hline \multicolumn{3}{|l|}{ Sexo } \\
\hline Feminino & 186 & 61,8 \\
\hline Masculino & 115 & 38,2 \\
\hline Total & 301 & 100,0 \\
\hline \multicolumn{3}{|l|}{ Faixa etária (anos) } \\
\hline $60-69$ & 129 & 42,9 \\
\hline $70-79$ & 119 & 39,5 \\
\hline $80-89$ & 43 & 14,3 \\
\hline$\geq 90$ & 10 & 3,3 \\
\hline Total & 301 & 100,0 \\
\hline \multicolumn{3}{|l|}{ Escolaridade } \\
\hline Analfabeto & 77 & 25,0 \\
\hline Ensino Fundamental incompleto & 130 & 43,1 \\
\hline Ensino Fundamental completo & 55 & 18,2 \\
\hline Ensino Médio & 28 & 9,3 \\
\hline Ensino Superior & 11 & 3,6 \\
\hline Total & 301 & 100,0 \\
\hline \multicolumn{3}{|l|}{ Condições de comunicação } \\
\hline Preservada & 268 & 89,6 \\
\hline Parcialmente comprometida & 25 & 8,3 \\
\hline Totalmente comprometida & 7 & 2,3 \\
\hline Total & 301 & 100,0 \\
\hline \multicolumn{3}{|l|}{ Estado marital } \\
\hline Vive com o companheiro & 190 & 63,1 \\
\hline Vive sem o companheiro & 111 & 36,9 \\
\hline Total & 301 & 100,0 \\
\hline \multicolumn{3}{|l|}{ Serviços de saúde que utiliza } \\
\hline Unidade de saúde da família & 188 & 62,4 \\
\hline Convênio & 58 & 19,2 \\
\hline Convênio/Unidade de saúde da família & 52 & 17,3 \\
\hline Total & 301 & 100,0 \\
\hline \multicolumn{3}{|l|}{ Com quem mora } \\
\hline Sozinho & 30 & 10,0 \\
\hline Companheiro apenas & 91 & 30,2 \\
\hline Filhos & 63 & 20,9 \\
\hline Companheiro/Filhos/Netos & 99 & 32,9 \\
\hline Amigos/Parentes & 18 & 6,0 \\
\hline Total & 301 & 100,0 \\
\hline \multicolumn{3}{|l|}{ Estado cognitivo } \\
\hline Normal & 224 & 74,4 \\
\hline Anormal & 77 & 25,6 \\
\hline Total & 301 & 100,0 \\
\hline \multicolumn{3}{|l|}{ Grau de dependência } \\
\hline Independente & 198 & 65,8 \\
\hline Dependente leve & 63 & 20,9 \\
\hline Dependente moderado & 25 & 8,3 \\
\hline Dependente severo & 14 & 4,7 \\
\hline Total & 301 & 100,0 \\
\hline
\end{tabular}

126 (16,9\%), e aquelas doenças endócrinas, nutricionais e metabólicas, 58 (7,7\%).

O número total de medicamentos utilizados pelos idosos foi 883, entre os quais se encontram aqueles cujos dados da literatura foram insuficientes para identificá-los e/ou classificálos. Citam-se, como exemplo, as fórmulas manipuladas e os fitoterápicos como "castanha da índia" e Ginkgo biloba, além de alguns nomes comerciais. Ainda assim, eles foram contabilizados ao se considerar o total de medicamentos utilizados. Em média, os idosos estudados utilizam 2,9 medicamentos/idoso.

Ao analisar os medicamentos utilizados pelos idosos, constatou-se que 450 (51\%) deles não se apresentavam com o nome genérico; além disso, 422 (47,6\%) não constavam na lista de medicamentos selecionados pela secretaria da saúde do município.

Na Tabela 3, observa-se que 43 (14,3\%) idosos afirmam que não estavam fazendo uso de medicamentos, já 89 (29,5\%) utilizam três ou quatro. Além de 12 (4\%) idosos que utilizam oito medicamentos ou mais.

Os medicamentos mais utilizados pelos idosos entrevistados foram: hipotensores, principalmente os inibidores da ECA, por 71 idosos; e os bloqueadores do canal de cálcio, por 33 idosos; diuréticos, como o hidrocorotiazida, utilizado por 65 idosos; antiagregante plaquetário, por 49 idosos; ansiolíticos, por 24 idosos; antidepressivo, por 36 deles; além dos hipoglicemiantes; antiinflamatórios não-esteróides; antisecretores e analgésicos, conforme Tabela 4.

Ressalta-se, na Tabela 5, que a maioria dos idosos, $214(71,1 \%)$, toma os medicamentos sozinhos; quase a metade, 134 (44,5\%), faz acompanhamento com mais de um médico; mais de um terço, 102 (33,9\%), compra os medicamentos e $83(27,5 \%)$ adquirem alguns em farmácia da rede pública e outros na rede privada. Dos idosos, 182 (60,5\%) afirmam que não esquecem de tomar os medicamentos e 45 (14,9\%) às vezes esquecem. Fazem uso de medicamentos por conta própria 111 (36,9\%) idosos e, além disso, $225(74,5 \%)$ afirmam ter recebido orientação quanto ao uso do medicamento e $246(81,7 \%)$ afirmam que sabem a indicação do mesmo, enquanto apenas $82(27,2 \%)$ dizem estar informados sobre os efeitos adversos dos medicamentos que utilizam.

Além disso, quando questionados se tinham alguma queixa em relação aos medicamentos, $180(59,8 \%)$ responderam que sim, sendo que muitos deles apontaram para a dificuldade em adquiri-los, devido ao alto custo. 


\section{Discussão}

Os dados do estudo foram obtidos a partir de $65,3 \%$ do universo elegível dos idosos e, apesar da representatividade, os resultados apresentados podem apresentar algum viés, principalmente, referindo-se à classe sócio-econômica, pois os idosos que aparentemente apresentavam melhores condições sócio-econômicas foram os que mais se recusaram a participar do estudo. Dados que apontam para o uso de maior quantidade de medicamentos entre idosos de renda mais alta foram encontrados em estudo de base populacional sobre o consumo de medicamentos entre idosos da cidade de Bambuí, Minas Gerais, Brasil 23 e em estudo na cidade do Rio de Janeiro, Brasil 24.

Entre outras limitações do estudo, há de se considerar as próprias de um estudo transversal e descritivo, tais como a falta de controle da qualidade das informações prestadas e de cruzamentos entre as variáveis. Mesmo assim, acredita-se que tais dados possam contribuir para a caracterização das condições de uso de medicamentos por idosos que vivem na comunidade estudada.

No presente estudo, as características sóciodemográficas dos idosos que vivem na área de abrangência de uma unidade do Programa Saúde da Família (PSF) indicam condições importantes para se proporem intervenções individuais e coletivas de utilização adequada de medicamentos.

Os dados apontam para a maior sobrevida das mulheres, sendo que elas representam $61,8 \%$ da população estudada, assim como em outros estudos 25 . No entanto, quando comparadas a homens da mesma idade, são consideradas mais vulneráveis a alterações no estado de saúde como quedas, múltiplas doenças, obesidade, pobreza, dependências diversas e, conseqüentemente, ao uso de múltiplos medicamentos 23 .

A baixa escolaridade dos idosos é considerada um sério fator de risco diante da complexidade dos esquemas medicamentosos utilizados pelos entrevistados, à medida que se constatou que $68,1 \%$ são analfabetos ou tiveram pouco tempo de escolaridade. Além disso, alguns deles apresentavam comprometimento nas condições de comunicação, outros moram sozinhos, 30 (10\%), o que deve ser foco da atenção dos profissionais de saúde que atendem tal parcela da população.

O estado cognitivo dos idosos estava comprometido em 77 (25,6\%) deles e 102 (33,9\%) tinham algum grau de dependência, o que compromete a autonomia para as atividades de vida diária, incluindo o uso correto de medicamentos.
Tabela 2

Distribuição dos diagnósticos médicos referidos pelos idosos entrevistados agrupados de acordo com a Classificação Internacional de Doenças, 10ạ revisão (CID-10) 22. Marília, São Paulo, Brasil, 2007.

\begin{tabular}{lcc}
\hline Diagnósticos referidos & $\mathbf{n}$ & $\%$ \\
\hline Doenças do aparelho circulatório & 328 & 44,0 \\
Doenças do sistema osteomuscular e do tecido conjuntivo & 126 & 16,9 \\
Doenças endócrinas nutricionais e metabólicas & 58 & 7,7 \\
Doenças do olho e anexos & 58 & 7,7 \\
Doenças do aparelho digestivo & 48 & 6,4 \\
Doenças do aparelho geniturinário & 32 & 4,3 \\
Doenças do aparelho respiratório & 27 & 3,6 \\
Doenças do sistema nervoso & 25 & 3,3 \\
Transtornos mentais e comportamento & 15 & 2,0 \\
Doenças da pele e tecidos subcutâneos & 12 & 1,6 \\
Neoplasias (tumores) & 10 & 1,3 \\
Outros & 7 & 0,9 \\
Total & 746 & 100,0 \\
\hline
\end{tabular}

Tabela 3

Distribuição percentual dos idosos de acordo com o número de medicamentos que utilizam. Marília, São Paulo, Brasil, 2007.

\begin{tabular}{lcc}
\hline $\begin{array}{l}\text { Número de medicamentos } \\
\text { utilizados por idoso }\end{array}$ & $\mathbf{n}$ & \\
\hline Nenhum & & \\
1 & 43 & 14,3 \\
2 & 48 & 15,9 \\
3 & 53 & 17,6 \\
4 & 52 & 17,3 \\
5 & 37 & 12,2 \\
6 & 25 & 8,3 \\
7 & 19 & 6,4 \\
8 & 12 & 4,0 \\
9 & 5 & 1,7 \\
10 & 5 & 1,7 \\
Total & 2 & 0,7 \\
\hline
\end{tabular}

Conforme retratado no estudo, o serviço de saúde que a maioria deles $188(62,4 \%)$ utiliza é a unidade de saúde da família. Esse número é acrescido de 52 (17,3\%) idosos que além dos serviços de assistência à saúde do convênio, também recebem atenção dos profissionais da unidade de saúde da família. Desta forma, amplia-se o papel da equipe, em especial frente à necessidade de responsabilizar-se pela adequada prescrição, 
Classes, subgrupos de medicamentos mais freqüentemente utilizados pelos idosos. Marília, São Paulo, Brasil, 2007.

\begin{tabular}{|c|c|c|}
\hline Classe medicamentosa & Medicamento & $\mathbf{n}$ \\
\hline \multicolumn{3}{|l|}{ Drogas que afetam o sistema cardiovascular } \\
\hline \multicolumn{3}{|l|}{ Hipotensores } \\
\hline Inibidor da ECA & Captopril & 71 \\
\hline Bloqueador do canal de cálcio & Nifedipina & 33 \\
\hline Bloqueador cardiosseletivo $\beta 1$ & Atenolol & 27 \\
\hline Betabloqueador & Propanolol & 14 \\
\hline \multicolumn{3}{|l|}{ Antiarrítmico (segundo classificação de Vaughan-Williams) } \\
\hline Antiarrítmico da classe III & Amiodarona & 12 \\
\hline \multicolumn{3}{|l|}{ Cardiotônico } \\
\hline Digitálicos & Digoxina & 9 \\
\hline \multicolumn{3}{|l|}{ Drogas que afetam os rins } \\
\hline Antagonista da aldosterona & Espironolactona & 4 \\
\hline \multirow[t]{3}{*}{ Diurético } & Furosemida & 3 \\
\hline & Hidroclorotiazida & 65 \\
\hline & Clortalidona & 3 \\
\hline \multicolumn{3}{|l|}{ Drogas utilizadas na homeostasia e trombose } \\
\hline Agentes antiplaquetários & AAS & 49 \\
\hline \multicolumn{3}{|l|}{ Drogas que afetam o pâncreas e sistema endócrino } \\
\hline Insulina exógena & Insulina & 5 \\
\hline \multicolumn{2}{|l|}{ potássio ATP-dependentes) } & 14 \\
\hline Biguanida & Metformina & 21 \\
\hline \multicolumn{3}{|l|}{ Drogas que afetam o sistema gastrointestinal } \\
\hline \multirow[t]{2}{*}{ Antagonista dos receptores $\mathrm{H} 2$ da histamina } & Ranitidina & 10 \\
\hline & Cimetidina & 8 \\
\hline Inibidor da bomba gástrica & Omeprazol & 9 \\
\hline \multicolumn{3}{|l|}{ Hipolipemiante } \\
\hline Inibidor da redutase HMG-CoA & Sinvastatina & 18 \\
\hline \multicolumn{3}{|l|}{ Drogas que afetam o sistema nervoso central } \\
\hline \multicolumn{3}{|l|}{ Agentes ansiolíticos } \\
\hline \multirow[t]{3}{*}{ Benzodiazepínicos } & Clonazepam & 8 \\
\hline & Diazepam & 7 \\
\hline & Alprazolam & 9 \\
\hline \multicolumn{3}{|l|}{ Antidepressivos } \\
\hline \multirow[t]{3}{*}{ Inibidor da recaptação da serotonina } & Fluoxetina & 16 \\
\hline & Sertralina & 3 \\
\hline & Citalopram & 1 \\
\hline \multirow[t]{3}{*}{ Tricíclicos } & Nortriptilina & 3 \\
\hline & Amitriptilina & 12 \\
\hline & Imipramina & 1 \\
\hline \multicolumn{3}{|l|}{ Drogas que atuam nos ossos } \\
\hline \multirow[t]{2}{*}{ Suplementos de cálcio } & Carbonato de cálcio & 7 \\
\hline & Alendronato de cálcio & 5 \\
\hline \multicolumn{3}{|l|}{ Analgésicos e substâncias correlatas } \\
\hline Analgésico antitérmico & & \\
\hline Analgésico não-opióides & Dipirona & 9 \\
\hline & Paracetamol & 15 \\
\hline Agentes antiinflamatórios & & \\
\hline Antiinflamatório não hormonal & Meloxicam & 5 \\
\hline & Diclofenaco sódico & 12 \\
\hline & Nimesulida & 11 \\
\hline & Cetoprofeno & 4 \\
\hline Antiinflamatório hormonal & & \\
\hline Esteróide & Budesonida & 5 \\
\hline
\end{tabular}


dispensação e utilização dos medicamentos pelos idosos e no incentivo à adoção de medidas não farmacológicas para o manejo dos problemas de saúde.

Outro aspecto bastante relevante, quando se considera o processo de envelhecimento, é a presença de múltiplas doenças. Os idosos apresentaram em média 2,5 diagnósticos referidos e, assim como em outros estudos, as doenças cardiovasculares foram aquelas que mais incidiram, seguidas das doenças do sistema osteomuscular e do tecido conjuntivo, além das doenças endócrinas, nutricionais e metabólicas. Dados referentes ao diagnóstico principal das internações hospitalares, entre idosos, mostram que as doenças do aparelho circulatório foram as mais freqüentes, seguidas das doenças do aparelho respiratório e do aparelho digestivo ${ }^{26}$. Muitas dessas enfermidades, por serem crônicas, demandam alto custo na assistência à saúde, além de propiciarem o surgimento de complicações com grande interferência no grau de dependência e qualidade de vida das pessoas.

Em conseqüência das múltiplas doenças, os idosos utilizam múltiplos medicamentos, sendo que a população estudada utiliza em média 2,9 medicamentos/idoso. Dados semelhantes foram encontrados em estudo com idosos atendidos em ambulatório de psicogeriatria, que consumiam três ou mais medicamentos por dia 27 . No presente estudo, apesar de alguns não consumirem medicamento, há aqueles que chegam a consumir diariamente oito a dez, chamando a atenção o fato de quase um terço dos idosos, 105 (34,8\%), ingerir quatro ou mais medicamentos. Estudo que avaliou a qualidade do uso de medicamentos em 634 idosas que freqüentavam a universidade aberta da terceira idade demonstrou que 52,7\% utilizavam de um a quatro medicamentos, $34,4 \%$ de cinco a dez 8 . Já no presente estudo, esses índices foram de $63,12 \%$ e $22,6 \%$ respectivamente.

Mesmo sendo componente importante da Política Nacional de Medicamentos 28, o uso de genéricos ainda está aquém de ser uma realidade entre os idosos estudados, uma vez que mais da metade dos medicamentos utilizados pelos 301 idosos foram adquiridos pelo nome comercial. Além disso, 49,2\% dos medicamentos não constavam na lista de medicamentos selecionados pela Secretaria Municipal de Saúde. Arrais et al. 29 observaram que $79 \%$ das especialidades farmacêuticas não estavam incluídas na Lista de Medicamentos Essenciais da OMS e 72,2\% não faziam parte da Relação Nacional de Medicamentos Essenciais (RENAME). Tais dados remetem à discussão sobre o "acesso ou excesso" que aponta para as dificuldades dos idosos em adquirir os medicamentos, ao mesmo tempo em que existe
Tabela 5

Distribuição dos idosos entrevistados de acordo com dados de adesão ao uso dos medicamentos que utiliza. Marília, São Paulo, Brasil, 2007.

\begin{tabular}{|c|c|c|}
\hline \multirow[t]{2}{*}{ Adesão ao uso de medicamentos } & \multicolumn{2}{|c|}{ Total } \\
\hline & $\mathbf{n}$ & $\%$ \\
\hline \multicolumn{3}{|l|}{ Como toma o medicamento } \\
\hline Sozinho & 214 & 71,1 \\
\hline Supervisionado & 20 & 6,6 \\
\hline Medicado por outra pessoa & 26 & 8,6 \\
\hline Não utiliza medicamentos/Não sabe informar & 41 & 13,6 \\
\hline Total & 301 & 100,0 \\
\hline \multicolumn{3}{|l|}{ Acompanhamento em mais de um médico } \\
\hline Sim & 134 & 44,5 \\
\hline Não & 167 & 55,5 \\
\hline Total & 301 & 100,0 \\
\hline \multicolumn{3}{|l|}{ Onde adquire medicamento } \\
\hline Compra & 102 & 33,9 \\
\hline Farmácia do serviço público & 75 & 24,9 \\
\hline Ambos & 83 & 27,5 \\
\hline Não utiliza medicamentos/Não sabe informar & 41 & 13,6 \\
\hline Total & 301 & 100,0 \\
\hline \multicolumn{3}{|c|}{ Queixa/Dificuldades relacionadas ao uso de medicamento } \\
\hline Sim & 180 & 59,8 \\
\hline Não & 80 & 26,6 \\
\hline Não utiliza medicamentos/Não sabe informar & 41 & 13,6 \\
\hline Total & 301 & 100,0 \\
\hline \multicolumn{3}{|l|}{ Esquece de tomar medicamentos } \\
\hline Não & 182 & 60,5 \\
\hline Às vezes & 45 & 14,9 \\
\hline Raramente & 15 & 5,0 \\
\hline Sempre & 18 & 6,0 \\
\hline Não utiliza medicamentos/Não sabe informar & 41 & 13,6 \\
\hline Total & 301 & 100,0 \\
\hline \multicolumn{3}{|l|}{ Uso de medicamentos por conta própria } \\
\hline $\operatorname{Sim}$ & 111 & 36,9 \\
\hline Não & 190 & 63,1 \\
\hline Total & 301 & 100,0 \\
\hline \multicolumn{3}{|l|}{ Orientação para o uso de medicamento } \\
\hline $\operatorname{Sim}$ & 225 & 74,7 \\
\hline Não & 35 & 11,6 \\
\hline Não utiliza medicamentos/Não sabe informar & 41 & 13,6 \\
\hline Total & 301 & 100,0 \\
\hline \multicolumn{3}{|l|}{ Conhecimento da indicação do medicamento } \\
\hline Sim & 246 & 81,7 \\
\hline Não & 14 & 4,6 \\
\hline Não utiliza medicamentos/Não sabe informar & 41 & 13,6 \\
\hline Total & 301 & 100,0 \\
\hline \multicolumn{3}{|l|}{ Conhecimento dos efeitos adversos } \\
\hline $\operatorname{Sim}$ & 82 & 27,2 \\
\hline Não & 178 & 59,1 \\
\hline Não utiliza medicamentos/Não sabe informar & 41 & 13,6 \\
\hline Total & 301 & 100,0 \\
\hline
\end{tabular}


a subutilização de classes terapêuticas ocasionadas por hábitos de prescrição não sustentados em critérios científicos e pelo notável consumo de novidades 30 .

O uso de medicamentos, embora benéfico em muitas situações, merece alguns cuidados especiais. Os medicamentos utilizados para problemas do sistema cardiovascular foram os mais prevalentes, em especial, os hipotensores como o inibidor da ECA e bloqueador do canal de cálcio. Tais fármacos são considerados responsáveis pelas maiores freqüências de interações e, conseqüentemente, de possíveis reações adversas a medicamento 9. Merecem destaque também os diuréticos tiazídicos, cuja indicação deve contar com criteriosa avaliação com a finalidade de evitá-los na presença de alguns sinais e sintomas bastante freqüentes entre os idosos 31 .

Mosegui et al. 9 identificaram, entre idosas, a presença de eventos relacionados ao uso de fármacos com uma freqüência de $63 \%$ nas idosas que utilizavam $\beta$-bloqueadores, $49,3 \%$ daquelas que utilizam inibidores da ECA, 11,8\% das que fazem uso de diuréticos e $44,4 \%$ das que utilizam antidepressivos.

Os agentes antidepressivos, assim como outros medicamentos que atuam no sistema nervoso central, são utilizados com freqüência pelos idosos estudados. Tais medicamentos, quando prescritos para esta faixa etária, requerem tratamento por período prolongado, dois anos ou mais, e a maior sensibilidade desses indivíduos aos efeitos adversos pode comprometer a sua adesão ou criar situações de risco 32 .

Entre os medicamentos causadores de iatrogenias, entre os idosos, destacam-se os digitálicos e antiinflamatórios não hormonais ${ }^{33}$. Quanto aos digitálicos, estudo entre idosos hospitalizados identificou que $27,6 \%$ faziam uso, com indicação avaliada como inadequada em 52,7\% dos casos 34 . Mesmo considerando serem populações com características diferentes, tais dados são divergentes dos encontrados no presente estudo, uma vez que apenas 9 (3\%) idosos utilizam digitálicos.

O uso de hipoglicemiante oral também não está livre de riscos 33 . A glibenclamida, utilizada por $14(4,6 \%)$ idosos, apesar de considerada bem tolerada, nesta faixa etária, predispõe à hipoglicemia, aumentando o risco de infarto agudo do miocárdio, acidente vascular cerebral e quedas 35

O fato de 134 (44,5\%) idosos fazerem acompanhamento com mais de um médico representa uma dificuldade adicional para a equipe da unidade de saúde da família em função dos conhecidos bloqueios de comunicação entre pro- fissionais e/ou serviços de saúde, mesmo quando existem mecanismos formais de referência e contra-referência.

Condições freqüentes entre os idosos tais como cronicidade das doenças, uso de múltiplos medicamentos, efeitos adversos, e a falta de uma adequada prescrição são fatores que podem contribuir para alterações negativas no estado de saúde dessa população, tudo isso agravado pela não-adesão ao uso de medicamentos.

Considerando-se que 46 (15,2\%) idosos necessitam de alguém para lhes ministrar os medicamentos, impõe-se, também, o cuidado na preparação do cuidador para a administração correta da medicação.

Quase 40\% dos idosos referem se esquecer de tomar medicamentos, às vezes ou sempre, deixando clara a necessidade de apoio e supervisão, visando ao seu uso correto. Dessa forma, evitamse os riscos à saúde, principalmente se forem considerados alguns tipos de medicamentos essenciais no controle das doenças e prevenção de complicações, bastante utilizados por eles, como os hipotensores, antiarrítmicos, antiagregante plaquetário, hipoglicemiantes.

Os dados do presente estudo também confirmam que a automedicação é uma prática comum, pois $111(36,9 \%)$ dos idosos utilizam algum tipo de fármaco sem a indicação médica. Uma avaliação mais precisa desse aspecto não foi realizada em função da grande quantidade de informação já obtida, no entanto, em estudo que avaliou o perfil de automedicação no Brasil 29, foi constatado que 52,6\% eram de valor intrínseco não elevado, 79\% não estavam incluídos na lista de medicamentos essenciais da OMS e 72,2\% não faziam parte da RENAME. Na revisão de estudos sobre automedicação entre idosos foram encontradas taxas de $12 \%$ a $44 \% 36$.

Por fim, apesar de um número expressivo dos idosos entrevistados afirmar conhecer a indicação dos medicamentos (81,7\%), 59,1\% informaram desconhecer seus efeitos adversos, o que reforça, ainda mais, a necessidade de educação em saúde.

\section{Conclusões}

Os dados do presente estudo corroboram indicações encontradas na literatura nacional e internacional em relação ao uso de medicamentos por pessoas vivendo a terceira idade, em particular a polifarmácia, a automedicação, o desconhecimento sobre indicações e possíveis efeitos colaterais, incluindo as complicações decorrentes das interações medicamentosas. Tais problemas, quando associados à baixa escolaridade, aos ren- 
dimentos incompatíveis com as necessidades básicas dos idosos, além de alterações no estado cognitivo e variados graus de dependência, dão a dimensão da complexidade que a denominada "rede básica" ou "atenção primária" está trabalhando atualmente tendo em vista o envelhecimento da população.

O fato é que o uso de medicamentos entre os idosos assume, cada vez mais, inegável importância como estratégia terapêutica para compensar as alterações sofridas com o processo de envelhecimento ou visando controlar doenças crônicas bastante freqüentes na terceira idade, mesmo que outras formas de cuidado sejam incorporadas pelas equipes. Nessa medida, o estudo reforça a importância de o uso racional dos medicamentos, em todas as suas dimensões, ser tomado como objeto de preocupação das equipes, dos gerentes e dos gestores dos serviços e sistemas de saúde, mesmo porque uma boa assistência farmacêutica, como componente essencial da atenção aos idosos, só poderá ser garantida a partir de uma melhor integração da prática dos vários profissionais e de modos mais solidários e compartilhados de se organizar o cuidado.

\section{Resumo}

O uso de múltiplos medicamentos é uma condição freqüente entre os idosos, que, apesar de necessária, na maioria das vezes, predispõe a riscos em relação aos efeitos adversos e à interação medicamentosa. No presente estudo foi proposto verificar as características do uso de medicamentos, entre os idosos, considerando o perfil sócio-demográfico, as classes medicamentosas mais utilizadas por eles e dados referentes à adesão. Trata-se de um estudo descritivo, realizado com 301 idosos da área de abrangência de um Programa Saúde da Família (PSF), por meio de um inquérito realizado no domicilio do idoso, seguindo roteiro previamente estruturado. Destaca-se, entre os idosos, o predomínio do sexo feminino, analfabetismo, alterações no estado cognitivo e dependências diversas. Apresentam, em média, 2,5 diagnósticos e utilizam 2,9 medicamentos/ idoso. Alguns dos medicamentos são considerados de risco para a faixa etária. Dados de adesão aos medicamentos revelam dependência na administração, automedicação, esquecimento e conhecimento deficiente. Considera-se necessário acompanhamento sistemático dos idosos que utilizam múltiplos medicamentos, incentivo a medidas não-farmacêuticas, com ênfase nas ações educativas para mudança no estilo de vida, contando com uma equipe multiprofissional.

Uso de Medicamentos; Idoso; Programa Saúde da Família

\section{Colaboradores}

M. J. S. Marin participou na coordenação da coleta de dados, descrição e análise dos resultados e considerações finais. L. C. O. Cecílio auxiliou na análise dos dados e considerações finais. A. E. W. U. F. Perez contribuiu com a classificação e análise dos resultados. F. Santella, C. B. A. Silva, J. R. Gonçalves Filho e L. C. Roceti colaboraram igualmente na coleta e agrupamento dos dados, busca de material bibliográfico e análise dos resultados.

\section{Agradecimentos}

Este artigo é parte do projeto Os Idosos Precisam de Cuidados Especiais: Uma Proposta de Planejamento em $P S F$, financiado pela Fundação de Amparo à Pesquisa do Estado de São Paulo (FAPESP). 


\section{Referências}

1. Terceira idade: dados estatísticos sobre idosos. http://www.saudeemmovimento.com.br/ conteudos/conteúdo_frame.asp?cod_noticia $=91$ (acessado em 26/Jun/2005).

2. Organização Mundial da Saúde. Envelhecimento ativo: uma política de saúde. Brasília: Organização Pan-Americana da Saúde; 2005.

3. Silva Jr. JB, Gomes FBC, Cezário AC, Moura L. Doenças e agravos não-transmissíveis: bases epidemiológicas. In: Rouquayrol MZ, Almeida Filho $\mathrm{N}$, organizadores. Epidemiologia \& saúde. 6a $\mathrm{Ed}$. Rio de Janeiro: Editora Medsi; 2003. p. 289-311.

4. Lima-Costa MF. Epidemiologia do envelhecimento no Brasil. In: Rouquayrol MZ, Almeida Filho N, organizadores. Epidemiologia \& saúde. 6a Ed. Rio de Janeiro: Editora Medsi; 2003. p. 499-513.

5. Barros JAC, Joany S. Anúncios de medicamentos em revistas médicas: ajudando a promover a boa prescrição? Ciênc Saúde Coletiva 2002; 7:891-8.

6. Stolley JM, Buckwalter KC, Fjordbak B, Bush S. Iatrogenesis in the elderly: drug related problems. J Gerontol Nurs 1991; 17:12-7.

7. Jonhson JA, Bootman JL. Drug-related morbidity and mortality: a cost-of-illness model. Arch Intern Med 1995; 155:1949-56.

8. Lima-Costa MF, Barreto SM, Giatti L. Condições de saúde, capacidade funcional, uso de serviços de saúde e gastos com medicamentos da população idosa brasileira: um estudo descritivo baseado na Pesquisa Nacional por Amostra de Domicílios. Cad Saúde Pública 2003; 19:735-43.

9. Mosegui GBG, Rozenfeld S, Veras RP, Vianna CMM. Avaliação da qualidade do uso de medicamentos em idosos. Rev Saúde Pública 1999; 33:437-44.

10. Palmieri DT. Clearing up the confusion: adverse effects of medications in the elderly. J Gerontol Nurs 1991; 17:32-5.

11. Marin MJS. Preparando o idoso para a alta hospitalar [Tese de Doutorado]. Ribeirão Preto: Escola de Enfermagem de Ribeirão Preto, Universidade de São Paulo; 1999.

12. Bertoldi AD, Barros AJD, Hallal PC, Lima RC. Utilização de medicamentos em adultos: prevalência e determinantes individuais. Rev Saúde Pública 2004; 38:228-38.

13. World Health Organization. The safety of medicines in public health programmes: pharmacovigilance an essential tool. Geneva: World Health Organization; 2006.

14. Silveira LMC, Ribeiro VMB. Grupo de adesão ao tratamento: espaço de "ensinagem" para profissionais de saúde e pacientes. Interface Comun Saúde Educ 2004; 9:91-104.

15. Matta R. A casa e a rua: espaço, cidadania, mulher e morte no Brasil. 4a Ed. Rio de Janeiro: Editora Guanabara Koogan; 1991.

16. Achutti A, Azambuja MIR. Doenças crônicas nãotransmissíveis no Brasil: repercussões do modelo de atenção à saúde sobre a seguridade social. Ciênc Saúde Coletiva 2004; 9:833-40.

17. Ramos LR, Perracini M, Rosa TE, Kalache A. Significance and management of disability among urban elderly residents in Brazil. J Cross Cult Gerontol 1993; 8:313-23.
18. Almeida OP, Almeida SA. Confiabilidade da versão brasileira da Escala de Depressão em Geriatria (GDS) versão reduzida. Arq Neuropsiquiatr 1999; 57:421-6.

19. Burguez-Diaz R. Adesão ao tratamento medicamentoso em pacientes idosos. In: Papaléo Netto M, organizador. Gerontologia: a velhice e o envelhecimento em visão globalizada. São Paulo: Editora Atheneu; 1996. p. 230-41.

20. Melo JMS, organizador. DEF 2005/06: dicionário de especialidades farmacêuticas. 34a Ed. Rio de Janeiro: Editora Publicações Científicas; 2005.

21. Secretaria Municipal de Higiene e Saúde de Marília. Relação de medicamentos padronizados. Marília: Secretaria Municipal de Higiene e Saúde de Marília; 2006.

22. Organização Mundial da Saúde. Classificação estatística internacional de doenças e problemas relacionados à saúde, 10a revisão. São Paulo: Centro Colaborador da OMS para a Classificação de Doenças em Português; 1995.

23. Loyola Filho AI, Uchoa E, Firmo JOA, Lima-Costa MF. Estudo de base populacional sobre o consumo de medicamentos entre idosos: Projeto Bambuí. Cad Saúde Pública 2005; 21:545-53.

24. Miralles MA, Kimberlin CL. Perceived access to care and medication use among ambulatory elderly in Rio de Janeiro, Brazil. Soc Sci Med 1998; 46:345-55.

25. Fundação Sistema Estadual de Análise de Dados. Esperança de vida aumenta e diferença entre gêneros diminui: queda de homicídios em jovens poupa vidas e explica avanço masculino. http://www. seade.gov.br/produtos/espvida/espvida_jan2006. pdf (acessado em 31/Mai/2007).

26. Loyola Filho AI, Matos DL, Giatti L, Afradique ME, Peixoto SV, Lima-Costa MF. Causas de internações hospitalares entre idosos brasileiros no âmbito do Sistema Único de Saúde. Epidemiol Serv Saúde 2004; 13:229-38.

27. Almeida OP, Ratto L, Garrido R, Tamai S. Fatores preditores e conseqüências clínicas do uso de múltiplas medicações entre idosos atendidos em um serviço ambulatorial de saúde mental. Rev Bras Psiquiatr 1999; 21:152-7.

28. Conselho Nacional de Saúde, Ministério de Saúde. Diretrizes para uma política de medicamentos genéricos. http://www.datasus.gov.br/conselho/ DOCUMENTOS/gen2.htm (acessado em 21/ Out/2007).

29. Arrais PSD, Coelho HLL, Batista MCDS, Carvalho RER, Righi RE, Arnau JM. Perfil da automedicação no Brasil. Rev Saúde Pública 1997; 31:71-7.

30. Rozenfeld S. Prevalência, fatores associados e mau uso de medicamentos entre os idosos: uma revisão. Cad Saúde Publica 2003; 19:717-24.

31. Miranda RD, Perrotti TC, Bellinazzi VR, Nobrega TM, Cnederoglo MS, Toniolo Neto J. Hipertensão arterial no idoso: peculiaridades na fisiopatologia, no diagnóstico e no tratamento. Rev Bras Hipertens 2002; 9:293-300.

32. Schneider LS, Olin JT. Efficacy of acute treatment for geriatric depression. Int Psychogeriatr 1995; 7 Suppl:7-25. 
33. Carvalho Filho ET, Souza MAR, Vaz CMK, Hojaij NSL, Yoshihara LAK. Iatrogenia no idoso. RBM Rev Bras Med 1996; 53:117-37.

34. Cunha UGV, Barbosa MT, Paradela EM, Carvalho FG. Uso de digital em idosos admitidos em unidade de geriatria de hospital geral. Arq Bras Cardiol 1998; 71:695-8.
35. Nasri F, Sá JR, Schmidt MLR. Hipoglicemiantes orais e insulinas. In: Gorzoni ML, Toniolo Neto J, organizadores. Terapêutica clínica no idoso. São Paulo: Editora Sarvier; 1995. p. 148-60.

36. Sayd JD, Figueiredo MC, Vaena MLHT. Automedicação na população idosa do núcleo de atenção ao idoso da UnATI-UERJ. Textos Envelhecimento 2000; 3:21-34.

Recebido em 17/Ago/2007

Versão final reapresentada em 13/Dez/2007

Aprovado em 21/Dez/2007 